\title{
Non-equivalence between Heisenberg XXZ spin chain and Thirring model
}

\author{
T. Fujita ${ }^{1}$, T. Kobayashi ${ }^{2}$, and M. Hiramoto ${ }^{3}$ \\ Department of Physics, Faculty of Science and Technology, \\ Nihon University, Tokyo, Japan \\ and \\ H. Takahashi ${ }^{4}$ \\ Laboratory of Physics, Faculty of Science and Technology, \\ Nihon University, Chiba, Japan
}

\begin{abstract}
The Bethe ansatz equations for the spin 1/2 Heisenberg XXZ spin chain are numerically solved, and the energy eigenvalues are determined for the anti-ferromagnetic case. We examine the relation between the XXZ spin chain and the Thirring model, and show that the spectrum of the XXZ spin chain is different from that of the regularized Thirring model.
\end{abstract}

PACS numbers: 11.10.Kk, 03.70.+k, 11.30.-j, 11.30.Rd

\footnotetext{
${ }^{1}$ e-mail: fffujita@phys.cst.nihon-u.ac.jp

${ }^{2}$ e-mail: tkoba@phys.cst.nihon-u.ac.jp

${ }^{3}$ e-mail: hiramoto@phys.cst.nihon-u.ac.jp

${ }^{4}$ e-mail: htaka@phys.ge.cst.nihon-u.ac.jp
} 


\section{Introduction}

The symmetry breaking has been discussed quite extensively in varieties of field theories $[1,2]$. It is believed that the spontaneous symmetry breaking should accompany a massless boson, and since there should not physically exist a massless boson in two dimensional filed theory, the symmetry breaking should not occur in two dimensional field theory model.

However, fermion field theory models are quite different in that bosons must be dynamically constructed by fermions and antifermions. For the fermion field theory models, the Goldstone boson has been known only for the current current interaction model of Nambu and Jona-Lasinio (NJL model) [3]. However, recent careful studies prove that there is no massless boson in the NJL model after the chiral symmetry breaking [4, 5]. The physics of the chiral symmetry breaking is rather simple. The chiral symmetry which is possessed in the NJL Lagrangian with massless fermion is broken in the new vacuum since the new vacuum is lower than the trivial one. In this case, the originally massless fermion acquires the finite mass, and becomes a massive NJL model which predicts always a massive boson, and the boson can never become massless since the induced fermion mass can never be set to zero.

In the same way, the chiral symmetry breaking in the massless Thirring model is broken in the new vacuum, but there appears no massless boson $[4,5]$.

In this Letter, we solve numerically the Bethe ansatz equations for the spin $1 / 2$ Heisenberg XXZ model $[6,7]$ and obtain the energy eigenvalues of the antiferromagnetic states. Since the massless Thirring model is believed to be equivalent to the spin $1 / 2$ Heisenberg XXZ model at the continuum limit [8], it should be interesting to compare the results of the energy eigenvalues of the two models at the continuum limit.

Here, we calculate the energy eigenvalues of the excited states for the spin $1 / 2$ Heisenberg XXZ model and for the regularized Thirring model. It turns out that they do not agree with each other. The regularized Thirring model has the first excited state with a finite gap while the XXZ spin chain has the gapless excitations. This indicates that the XXZ spin chain and the regularized Thirring model are not equivalent to each other since physical observables are different.

Here, we discuss the physics behind the difference between the two models. The equivalence between the spin 1/2 Heisenberg XYZ model and the massive Thirring model is well established [8]. But the massless limit in the massive Thirring model is a singular 
point and should not be taken naively. The massless Thirring has two vacua, one which corresponds to the trivial vacuum with the chiral symmetry preserved, and the other which is a true vacuum with the violation of the chiral symmetry. One sees that the true vacuum state is lower than the trivial one, and therefore the true vacuum is physically realized. From the present analysis, we show that the XXZ spin chain cannot be reduced to the Thirring model with the true vacuum even though one may mathematically obtain the Thirring Lagrangian from the XXZ spin chain.

The proof for the equivalence between the XXZ spin chain and the Thirring model is based on the naive continuum limit of the XXZ model. However, the XXZ model has only one scale, and therefore, the physical meaning of the continuum limit is not clear. All the physical observables are measured by the lattice constant $a$, and thus in order that the very small a makes sense, one should compare $a$ with other scale quantity. In this respect, the continuum limit of the XYZ can be well defined, but the XXZ model should keep the lattice constant $a$ finite. Even if one says that one could derive the field theory model at the continuum limit ( small lattice constant $a$ ), all the observables of this field theory model should be measured by the lattice constant $a$. In the Thirring Lagrangian derived mathematically from the XXZ spin chain, there is no scale parameter corresponding to the lattice constant $a$, and physically, this indicates that the XXZ spin chain and the Thirring model must be different from each other.

This paper is organized in the following way. In the next section, we briefly explain

the Bethe ansatz solutions for the XXZ spin chain. Section 3 treats the massless Thirring model, and the energy eigenvalues of the vacuum and the excited states with the chiral symmetry breaking are discussed. In section 4 , we discuss the equivalence between the XXZ spin chain and the massless Thirring models. Section 5 summarizes what we have learned here.

\section{Heisenberg XXZ model}

Here, we briefly describe the Heisenberg XXZ model. The XXZ model has the following Hamiltonian $[6,7]$

$$
H=J \sum_{i=1}^{N}\left(S_{i}^{x} S_{i+1}^{x}+S_{i}^{y} S_{i+1}^{y}+\Delta S_{i}^{z} S_{i+1}^{z}\right)
$$

where $S_{i}^{a}$ is a spin operator at the site $i . J$ and $\Delta$ denote the coupling constant and the anisotropy parameter, respectively, and $N$ is the site number. The periodicity $S_{N+i}=S_{i}$ 
is assumed. This Hamiltonian can be numerically solved by the exact diagonalization. However, if one wants to discuss the excitation spectrum, then one has to have the site number $N$ which must be larger than $N=1000$ or so [9]. This is practically impossible.

Fortunately, this model is solved by the Bethe ansatz technique, and the Hamiltonian can be diagonalized by the superposition of the wave functions $\phi\left(z_{n_{1}}, \ldots, z_{n_{m}}\right)$ for $m$ down spin case as

$$
\Psi=\sum_{P} A\left(n_{1}, \ldots, n_{m}\right) \phi\left(z_{n_{1}}, \ldots, z_{n_{m}}\right)
$$

where $P$ means all possible permutations of the $n_{1}, \ldots, n_{m}$. Further, the coefficient $A\left(n_{1}, \ldots, n_{m}\right)$ is assumed to be of the following shape,

$$
A\left(n_{1}, \ldots, n_{m}\right)=\sum_{P_{\mu}} \sum_{P} \exp \left(i \sum_{j}^{m} k_{P_{j}} n_{\mu_{j}}+\frac{1}{2} \sum_{j<\ell} \varphi_{P_{j} P_{\ell}}\right)
$$

where $k_{i}$ denote the pseudo-momentum of the down-spin site. From the periodic boundary conditions, we obtain the following equations

$$
N k_{j}=2 \pi \lambda_{j}+\sum_{\ell} \varphi_{j \ell}
$$

where $\lambda_{i}$ are integers running between 0 and $N-1$ with the condition of $\lambda_{1} \leq \lambda_{2} \leq \ldots \leq$ $\lambda_{m}$. The equation for $\varphi_{j \ell}$ becomes

$$
\cot \frac{\varphi_{j \ell}}{2}=\frac{\Delta \sin \left(\frac{k_{j}-k_{\ell}}{2}\right)}{\cos \left(\frac{k_{j}+k_{\ell}}{2}\right)-\Delta \cos \left(\frac{k_{j}-k_{\ell}}{2}\right)}
$$

In this case, we can express the energy eigenvalue $E$ as

$$
E=\left(\frac{1}{4} N-m\right) \Delta+\sum_{j=1}^{m} \cos k_{j} .
$$

The Bethe ansatz equations (2.5) can be numerically solved by the new iteration method which is developed in ref. [10, 11].

Here, we should write the translation of the coupling constants between the spin chain and the Thirring model [8], and the Thirring coupling constant $g$ is related to the $\Delta$ as

$$
g=\frac{4 \pi \Delta}{2 \pi-\Delta} .
$$

Here, it should be noted that the correspondence between the two models is only meaningful for the condition

$$
\Delta \leq \frac{2}{5} \pi
$$


since $g$ must be smaller than $\pi$ [8]. The vacuum state of the field theory corresponds to the state of $S_{z}=0$, which is just the anti-ferromagnetic state. In section 4 , the numerical results of the excitation spectrum will be discussed.

Here, we briefly describe the procedure commonly employed to obtain the Thirring model Lagrangian from eq.(2.1) [12]. By the Jordan-Wigner transformation, one can rewrite the Hamiltonian of eq.(2.1) in terms of the spinless lattice fermion

$$
H=J \sum_{i=1}^{N}\left[\frac{1}{2}\left(\psi_{i}^{\dagger} \psi_{i+1}+\text { h.c. }\right)+\Delta\left(\psi_{i}^{\dagger} \psi_{i}-\frac{1}{2}\right)\left(\psi_{i+1}^{\dagger} \psi_{i+1}-\frac{1}{2}\right)\right]
$$

This Hamiltonian can be reduced to the massless Thirring model Lagrangian below when one takes naively the continuum limit [12].

\section{Thirring model}

The massless Thirring model is described by the following Lagrangian density [13]

$$
\mathcal{L}=i \bar{\psi} \gamma_{\mu} \partial^{\mu} \psi-\frac{1}{2} g j^{\mu} j_{\mu}
$$

where the fermion current $j_{\mu}$ is given as $j_{\mu}=: \bar{\psi} \gamma_{\mu} \psi:$.

This model is studied by the Bogoliubov transformation, and it is found that the vacuum has a chiral symmetry broken phase $[4,5,14,15]$. The vacuum energy $E_{v a c}$ as measured from the trivial vacuum $\left(E_{v a c}=0\right)$ is given

$$
E_{v a c}=-\frac{L}{2 \pi} \frac{\Lambda^{2}}{\sinh \left(\frac{\pi}{g}\right)} e^{-\frac{\pi}{g}}
$$

where $\Lambda$ and $L$ denote the cutoff momentum and box length in this model, respectively, and all of the physical quantities must be measured by the $\Lambda$.

Now, in order to compare directly the regularized Thirring model prediction to the energy eigenvalues of the XXZ spin chain model, we start the equation for the boson in the regularized Thirring model where we still keep the box length $L$ finite. The equation for the boson wave function becomes just the same as the massive Thirring model [16] and can be written as $[4,5]$

$$
E f_{n}=2 E_{p_{n}} f_{n}-\frac{g}{L} \sum_{l=-N_{0}}^{N_{0}} f_{l}\left(1+\frac{M^{2}}{E_{p_{n}} E_{p_{l}}}+\frac{p_{n} p_{l}}{E_{p_{n}} E_{p_{l}}}\right)
$$


where $p_{n}$ and $E_{p_{n}}$ are given as

$$
p_{n}=\frac{2 \pi}{L} n, \quad E_{p_{n}}=\sqrt{M^{2}+p_{n}^{2}} .
$$

Further, the induced fermion mass $M$ is given as the solution of the following equation

$$
\frac{g}{L} \sum_{n=-N_{0}}^{N_{0}} \frac{1}{\sqrt{M^{2}+p_{n}^{2}}}=1 .
$$

$N_{0}$ is related to the cutoff momentum $\Lambda$ as

$$
\Lambda=\frac{2 \pi}{L} N_{0}
$$

In order to connect the present calculation with the spin chain, we write the box length $L$ in terms of the lattice spacing constant $a$ as

$$
L=N a
$$

with $N=2 N_{0}+1$. Thus, for the large $N$, we obtain

$$
\Lambda=\frac{\pi}{a} .
$$

Eq.(3.3) can be easily solved by defining $A$ and $B$ as $[16,17]$

$$
\begin{gathered}
A=\sum_{n=-N_{0}}^{N_{0}} f_{n} \\
B=\sum_{n=-N_{0}}^{N_{0}} \frac{f_{n}}{E_{n}} .
\end{gathered}
$$

In this case, we obtain $f_{n}$ as

$$
f_{n}=\frac{g}{L} \frac{A+\frac{M^{2}}{E_{n}} B}{2 E_{n}-E} .
$$

Putting $f_{n}$ into eqs.(3.9), we obtain the eigenvalue equation for $E$, and this can be solved in a straightforward manner.

At this point, we make a comment on the Bethe ansatz solution of the massless Thirring model by the technique developed by Bergknoff and Thacker [18]. This method can be applied if one introduces the cutoff momentum, and, in this case, the solution of [18] becomes quite similar to the solution obtained by the massive Thirring model and therefore one obtains the spectrum similar to fig. 2 [10]. In this sense, one has to introduce 


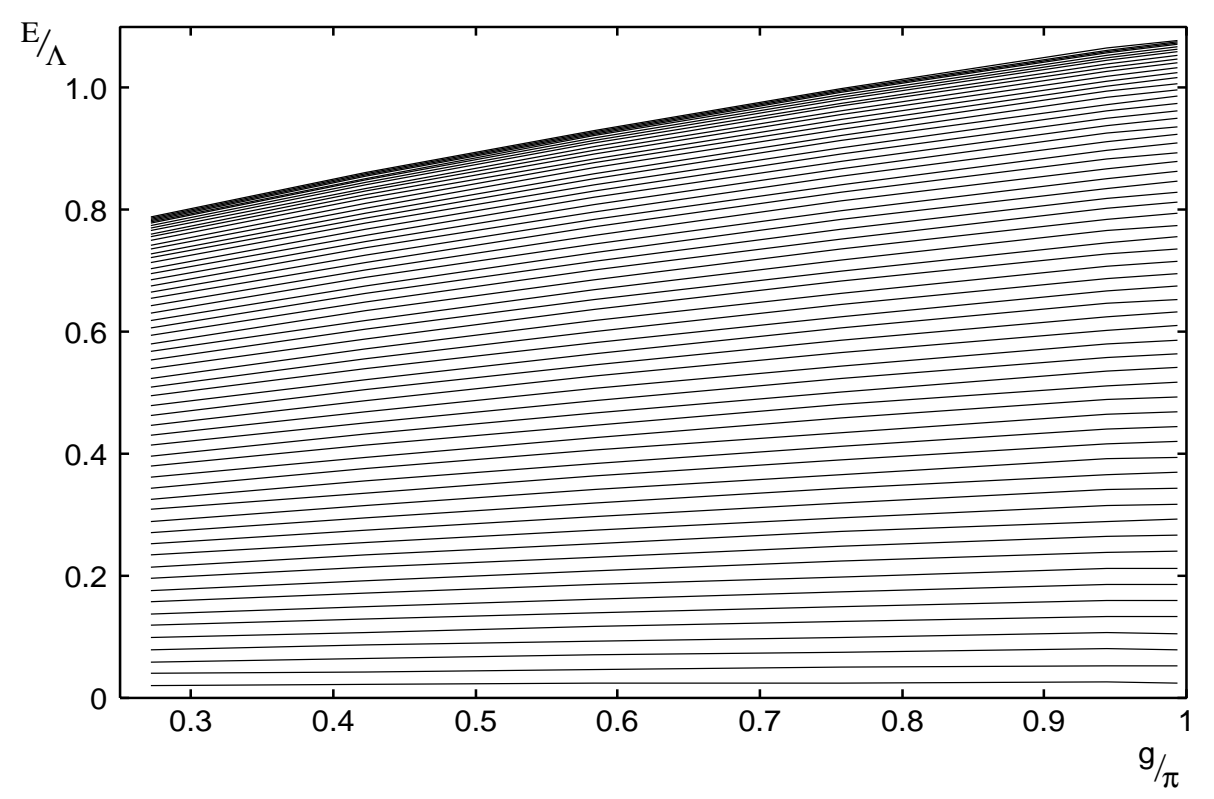

Figure 1: The calculated spectrum of the XXZ spin chain

a scale when one wants to describe the spectrum of the bosonic states in the Thirring model.

\section{Non-equivalence between Heisenberg XXZ and Thirring model}

The Heisenberg XYZ spin chain is known to be equivalent to the massive Thirring model at the continuum limit [8]. The translation of the coupling constants between XYZ and massive Thirring is given in eq.(2.7).

From the above equivalence between the XYZ spin chain and the massive Thirring models, one also expects the equivalence between the XXZ and the massless Thirring models since the XXZ spin chain corresponds to the massless limit of the XYZ spin chain. However, the massless limit is a singular point in the massive Thirring model, and therefore it is nontrivial whether the XXZ spin chain and the massless Thirring model are equivalent to each other. In particular, the massless Thirring model has no scale, and therefore, one has to introduce the cutoff momentum $\Lambda$ by which all of the observables 


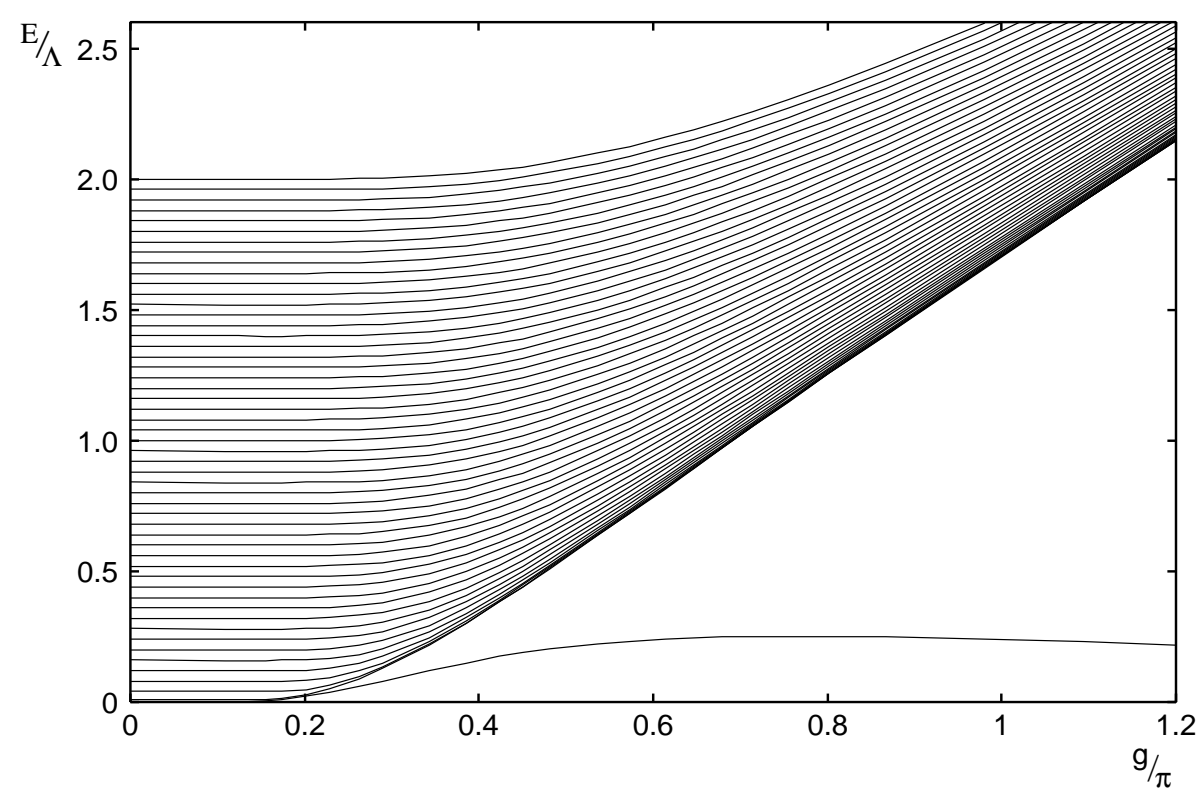

Figure 2: The predicted spectrum of the regularized Thirring model

must be measured. On the other hand, the XXZ spin chain has a natural scale of the lattice constant, and this is an important contrast to the massless Thirring model.

In figs. 1 and 2, we show the excitation spectrum of the two models. Fig. 1 shows the calculated result of the XXZ spin chain while fig. 2 shows the predicted spectrum of the regularized Thirring model. As can be seen from these figures, there is a significant difference between them. In the XXZ spin chain, there is no gap in the first excited state, but the regularized Thirring model has a finite gap in the first excited state. This means that the two models are not equivalent to each other, even though it is believed that the XXZ spin chain at the continuum limit corresponds to the massless Thirring model [12] if one considers the excitations near the fermi sea.

What is wrong with the derivation of the Thirring model from eq.(2.9) ? Here, we present our interpretation of the non-equivalence of the two models. In the XYZ spin chain, one can make a continuum limit since there are two parameters which have the dimensions, the lattice constant and the mass parameter. Therefore, one can make the proper continuum limit in the XYZ spin chain. However, when one makes a massless limit from the XYZ to XXZ, then the XXZ possesses only one scale, the lattice constant. In this case, one cannot take the continuum limit since everything is already measured by the lattice constant. The equivalence between the XXZ spin chain and the massless Thirring model derived up to now must be due to the improper procedure of the continuum limit 
in the XXZ spin chain. In this respect, one should say that there is no corresponding field theory of the XXZ spin chain in the continuum limit, and therefore it does not correspond to the massless Thirring model.

In fact, this continuum field theory of the massless Thirring model possesses the chiral symmetry which is not shared by the XXZ spin chain. This continuous symmetry plays a very important role for the vacuum structure. In the massless Thirring model, there are two vacua, one which preserves the chiral symmetry, and the other which violates the chiral symmetry. Under the chiral symmetry breaking, the true vacuum goes to the one which is lower than the trivial vacuum. In this respect, the physical vacuum of the massless Thirring model is the one that violates the chiral symmetry.

\section{Conclusions}

We have examined the relation between the spin 1/2 Heisenberg XXZ model and the massless Thirring model. It turns out that the spectrum of the XXZ model is different from the massless Thirring model, and it does not possess the true vacuum of the massless Thirring model. In this respect, the equivalence between the XXZ and the Thirring models does not hold, contrary to the case of the massive theory in which the XYZ spin chain is indeed equivalent to the massive Thirring model at the continuum limit.

This is essentially due to the fact that the massless Thirring model has the continuous symmetry (chiral symmetry) while the XXZ spin chain does not possess such a symmetry. Therefore, in the massless Thirring model, there are two vacua, one which keeps the chiral symmetry, and the other which violates the chiral symmetry. The true vacuum is the one that violates the chiral symmetry since it is lower than the other. But the XXZ spin chain cannot reproduce the true vacuum state of the massless Thirring model. 


\section{References}

[1] J. Goldstone, Nuovo Cimento, 19, 154 (1961)

[2] J. Goldstone, A. Salam and S. Weinberg, Phys. Rev. 127, 965 (1962).

[3] Y. Nambu and G. Jona-Lasinio, Phys. Rev. 122, 345 (1961)

[4] M. Hiramoto and T. Fujita, "No massless boson in chiral symmetry breaking in NJL and Thirring model", hep-th/0306083

[5] T. Fujita, M. Hiramoto and H. Takahashi, "No Goldstone boson in NJL and Thirring model", hep-th/0306110

[6] H.A. Bethe, Z. Phys. 71, 205 (1931).

[7] R. Orbach, Phys. Rev. 112, 309 (1958).

[8] A. Luther, Phys. Rev. B14, 2153 (1976).

[9] T. Fujita, T. Kobayashi and H. Takahashi, Phys. Rev. D in press, hep-th/0306173

[10] T. Fujita, Y. Sekiguchi and K. Yamamoto, Ann. Phys. 255, 204 (1997)

[11] T. Fujita, T. Kobayashi and H. Takahashi, J. Phys. A36,1553 (2003)

[12] I. Affleck, Les Houches, Session XLIX, 1990, North-Holland.

[13] W. Thirring, Ann. Phys. (N.Y) 3, 91 (1958).

[14] M. Faber and A.N. Ivanov, Eur. Phys. J. C20, 723 (2001).

[15] S. Coleman, Comm. Math. Phys. 31, 259 (1973)

[16] M. Hiramoto and T. Fujita, Phys. Rev. D 66, 045007 (2002).

[17] T. Fujita and A. Ogura, Prog. Theor. Phys. 89, 23 (1993).

[18] H. Bergknoff and H.B. Thacker, Phys. Rev. Lett. 42, 135 (1979) 